\title{
The Minimum Hub Distance Energy of a Graph
}

\author{
Veena Mathad \\ Department of Studies in Mathematics \\ University of Mysore, Manasagangotri \\ Mysuru - 570 006, INDIA
}

\author{
Sultan Senan Mahde \\ Department of Studies in Mathematics \\ University of Mysore, Manasagangotri \\ Mysuru - 570 006, INDIA
}

\begin{abstract}
In this paper, the concept of minimum hub distance energy $E_{H d}(G)$ of a connected graph $G$ is introduced and minimum hub distance energies of some standard graphs and a number of wellknown families of graphs are computed. Upper and lower bounds for $E_{H d}(G)$ are also established.
\end{abstract}

\section{Keywords}

Minimum hub set, minimum hub distance matrix, minimum hub distance eigenvalues, minimum hub distance energy.

AMS Subject Classification: 05C50, 05C99, $11 \mathrm{C08}$.

\section{INTRODUCTION}

In this paper, a simple graph $G=(V, E)$, that is nonempty, finite, having no loops, no multiple and directed edges are considered. Let $p$ and $q$ be the number of its vertices and edges, respectively. The symbols $\Delta(G)$ and $\delta(G)$ denote the maximum degree and the minimum degree of $G$, respectively. For graph theoretic terminology, we refer to [12].

M. Walsh [18] introduced the theory of hub numbers in the year 2006. Suppose that $H \subseteq V(G)$ and let $x, y \in V(G)$. An $H$-path between $x$ and $y$ is a path where all intermediate vertices are from $H$. (This includes the degenerate cases where the path consists of the single edge $x y$ or a single vertex $x$ if $x=y$, call such an $H$-path trivial). A set $H \subseteq V(G)$ is a hub set of $G$ if it has the property that, for any $x, y \in V(G)-H$, there is an $H$-path in $G$ between $x$ and $y$. The smallest size of a hub set in $G$ is called the hub number of $G$, and is denoted by $h(G)$ [18]. For more details on the hub number see [7]. A set $S \subseteq V(G)$ is called a dominating set of $G$ if each vertex of $V-S$ is adjacent to at least one vertex of $S$. The domination number of a graph $G$ denoted as $\gamma(G)$ is the minimum cardinality of a dominating set in $G$ [13].

The concept of energy of a graph was introduced by I. Gutman [9] in the year 1978. Let $G$ be a graph with $p$ vertices and $q$ edges and let $A=\left(a_{i j}\right)$ be the adjacency matrix of the graph. The eigenvalues $\lambda_{1}, \lambda_{2}, \ldots, \lambda_{p}$ of $\mathrm{A}$, assumed in non increasing order, are the eigenvalues of the graph $G$. As $A$ is real symmetric, the eigenvalues of $G$ are real with sum equal to zero. The energy $E(G)$ of $G$ is defined to be the sum of the absolute values of the eigenvalues of $G$, i.e. $E(G)=\Sigma_{i=1}^{p}\left|\lambda_{i}\right|$. For more details on the mathematical aspects of the theory of graph energy see [2, 10, 16].
The distance matrix of $G$ is the square matrix $A_{d}(G)$ of order $p$ whose $(i, j)$ - entry is the distance between the vertices $v_{i}$ and $v_{j}$. Let $\rho_{1}, \rho_{2}, \ldots, \rho_{p}$ be the eigenvalues of the distance matrix $A_{d}(G)$ of $G$. The distance energy $D E(G)$ of a graph $G$ is defined by

$$
D E(G)=\sum_{i=1}^{p}\left|\rho_{i}\right| .
$$

More detailed studies on distance energy can be found in [5, 6] 11 , 14. 17. Motivated by this definition, we introduce the concept of minimum hub distance energy $E_{H d}(G)$ of a graph $G$ and compute minimum hub energies of some standard graphs. Upper and lower bounds for $E_{H d}(G)$ are also established. It is possible that the minimum hub distance energy considered in this paper may have some applications in Chemistry as well as in other areas.

\section{THE MINIMUM HUB DISTANCE ENERGY OF A GRAPH}

Let $G$ be a graph of order $p$ with vertex set $V=\left\{v_{1}, v_{2}, \ldots, v_{p}\right\}$ and edge set $E$. Any hub set $H$ of a graph $G$ with minimum cardinality is called a minimum hub set. Let $H$ be a minimum hub set of a graph $G$. The minimum hub distance matrix of $G$ is the $p \times p$ matrix $A_{H d}(G)=\left(a_{i j}\right)$, where

$$
a_{i j}= \begin{cases}1, & \text { if } i=j \text { and } v_{i} \in H ; \\ d\left(v_{i}, v_{j}\right), & \text { otherwise. }\end{cases}
$$

The characteristic polynomial of $A_{H d}(G)$ denoted by $f_{p}(G, \rho)$, is defined as

$$
f_{p}(G, \rho):=\operatorname{det}\left(\rho I-A_{H d}(G)\right) .
$$

The minimum hub distance eigenvalues of the graph $G$ are the eigenvalues of $A_{H d}(G)$. Since $A_{H d}(G)$ is real and symmetric, its eigenvalues are real numbers and we label them in non-increasing order $\rho_{1} \geq \rho_{2} \geq \ldots \geq \rho_{p}$. The minimum hub distance energy of $G$ is defined as:

$$
E_{H d}(G)=\sum_{i=1}^{p}\left|\rho_{i}\right|
$$

EXAMPLE 1. Let $G$ be a graph in Figure 1 with vertices $\left\{v_{1}, v_{2}, v_{3}, v_{4}, v_{5}\right\}$ and consider its minimum hub set be $H_{1}=$ $\left\{v_{2}, v_{3}\right\}$. 


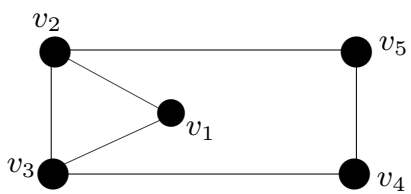

Figure 1

Then

$$
A_{H_{1} d}(G)=\left(\begin{array}{lllll}
0 & 1 & 1 & 2 & 2 \\
1 & 1 & 1 & 2 & 1 \\
1 & 1 & 1 & 1 & 2 \\
2 & 2 & 1 & 0 & 1 \\
2 & 1 & 2 & 1 & 0
\end{array}\right)
$$

The characteristic polynomial of $A_{H_{1} d}(G)$ is $f_{p}(G, \rho)=\rho^{5}-$ $2 \rho^{4}-21 \rho^{3}-20 \rho^{2}+11 \rho+6$, the minimum hub distance eigenvalues are $\rho_{1} \approx 6, \rho_{2} \approx 0.61803, \rho_{3} \approx-0.38197, \rho_{4} \approx-1.618, \rho_{5} \approx$ -2.618 . Therefore the minimum hub distance energy of $G$ is

$$
E_{H_{1} d}(G) \approx 11.236 \text {. }
$$

If we take another minimum hub set of $G$, namely $H_{2}=\left\{v_{1}, v_{2}\right\}$, then

$$
A_{H_{2} d}(G)=\left(\begin{array}{ccccc}
1 & 1 & 1 & 2 & 2 \\
1 & 1 & 1 & 2 & 1 \\
1 & 1 & 0 & 1 & 2 \\
2 & 2 & 1 & 0 & 1 \\
2 & 1 & 2 & 1 & 0
\end{array}\right)
$$

The characteristic polynomial of $A_{H_{2} d}(G)$ is $f_{p}(G, \rho)=\rho^{5}-$ $2 \rho^{4}-21 \rho^{3}-23 \rho^{2}+6 \rho+3$, the minimum hub distance eigenvalues are $\rho_{1} \approx 6.0614, \rho_{2} \approx 0.41421, \rho_{3} \approx-0.27744, \rho_{4} \approx$ $-1.7840, \rho_{5} \approx-2.4142$. Therefore the minimum hub distance energy of $G$ is

$$
E_{H_{2} d}(G) \approx 10.951 \text {. }
$$

The above example illustrates that the minimum hub distance energy of a graph $G$ depends on the choice of the minimum hub set. i.e., the minimum hub distance energy is not a graph invariant. We need the following to prove main results.

THEOREM 1. [15] For any $(p, q)$ graph $G, p-q \leq \gamma(G)$. Furthermore, $\gamma(G)=p-q$ if and only if each component of $G$ is a star.

LEMMA 2. [18] For any graph $G, \gamma(G) \leq h(G)+1$.

THEOREM 3. [18] If $G$ is a connected graph then $h(G) \leq$ $|V(G)|-\Delta(G)$, and the inequality is sharp.

\section{SOME BASIC PROPERTICES OF MINIMUM HUB DISTANCE ENERGY}

THEOREM 4. Let $G$ be a graph of order p, size $q$, and hub number $h(G)$. Let $f_{p}(G, \rho)=c_{0} \rho^{p}+c_{1} \rho^{p-1}+c_{2} \rho^{p-2}+\ldots+c_{p}$ be the characteristic polynomial of minimum hub distance matrix of a graph $G$. Then

(1) $c_{0}=1$.

(2) $c_{1}=-h(G$.

(3) $c_{2}=\left(\begin{array}{c}h(G) \\ 2\end{array}\right)-\sum_{1 \leq i<j \leq p} d^{2}\left(v_{i}, v_{j}\right)$
Proof. (1) Follows by the definition of $f_{p}(G, \rho)$.

(2) Since the sum of diagonal elements of $A_{H d}(G)$ is equal to $|H|=h(G)$, where $H$ is a minimum hub set of a graph $G$. The sum of determinants of all $1 \times 1$ principal submatrices of $A_{H d}(G)$ is the trace of $A_{H d}(G)$, which evidently is equal to $h(G)$. Thus, $(-1)^{1} c_{1}=h(G)$.

(3) $(-1)^{2} c_{2}$ is equal to the sum of determinants of all $2 \times 2$ principal submatrices of $A_{H d}(G)$, that is

$$
\begin{aligned}
c_{2} & =\sum_{1 \leq i<j \leq p}\left|\begin{array}{cc}
a_{i i} & a_{i j} \\
a_{j i} & a_{j j}
\end{array}\right| \\
& =\sum_{1 \leq i<j \leq p}\left(a_{i i} a_{j j}-a_{i j} a_{j i}\right) \\
& =\sum_{1 \leq i<j \leq p} a_{i i} a_{j j}-\sum_{1 \leq i<j \leq p} a_{i j}^{2} \\
& =\left(\begin{array}{c}
h(G) \\
2
\end{array}\right)-\sum_{1 \leq i<j \leq p} d^{2}\left(v_{i}, v_{j}\right) .
\end{aligned}
$$

THEOREM 5. Let $G$ be a graph of order p. Let $\rho_{1}, \rho_{2}, \ldots, \rho_{p}$ be the eigenvalues of $A_{H d}(G)$. Then

$$
\begin{aligned}
& \text { (i) } \sum_{i=1}^{p} \rho_{i}=h(G) . \\
& \text { (ii) } \sum_{i=1}^{p} \rho_{i}^{2}=h(G)+2 q+2 D \text {, where } D= \\
& \sum_{i<j, d\left(v_{i}, v_{j}\right) \neq 1} d^{2}\left(v_{i}, v_{j}\right) .
\end{aligned}
$$

Proof. (i) Since the sum of eigenvalues of $A_{H d}(G)$ is the trace of $A_{H d}(G)$, then

$\sum_{i=1}^{p} \rho_{i}=\sum_{i=1}^{p} a_{i i}=|H|=h(G)$, where $H$ is a minimum hub set of $G$.

(ii) Similarly, the sum of squares of the eigenvalues of $A_{H d}(G)$ is the trace of $\left(A_{H d}(G)\right)^{2}$. Then

$$
\begin{aligned}
\sum_{i=1}^{p} \rho_{i}^{2} & =\sum_{i=1}^{p} \sum_{j=1}^{p} a_{i j} a_{j i} \\
& =\sum_{i=1}^{p} a_{i i}^{2}+\sum_{i \neq j}^{p} a_{i j} a_{j i} \\
& =\sum_{i=1}^{p} a_{i i}^{2}+2 \sum_{i<j}^{p} a_{i j}^{2} \\
& =|H|+2 \sum_{1 \leq i<j \leq p} d^{2}\left(v_{i}, v_{j}\right) \\
& =h(G)+2 \sum_{1 \leq i<j \leq p} d^{2}\left(v_{i}, v_{j}\right) \\
& =h(G)+2 q+2 D, \text { where } D=\sum_{i<j, d\left(v_{i}, v_{j}\right) \neq 1} d^{2}\left(v_{i}, v_{j}\right) .
\end{aligned}
$$

LEMMA 6. Let $G$ be a graph with a minimum hub set $H$. If the minimum hub distance energy $E_{H d}(G)$ of $G$ is a rational number, 
then

$$
E_{H d}(G) \equiv|H|(\bmod 2) .
$$

PROOF. Let $\rho_{1}, \rho_{2}, \ldots, \rho_{p}$ be minimum hub distance eigenvalues of a graph $G$ of which $\rho_{1}, \rho_{2}, \ldots, \rho_{r}$ are positive and the remaining are non-positive, then

$$
\begin{aligned}
\sum_{i=1}^{p}\left|\rho_{i}\right| & =\left(\rho_{1}+\rho_{2}+\ldots+\rho_{r}\right)-\left(\rho_{r+1}+\rho_{r+2}+\ldots+\rho_{p}\right) \\
& =2\left(\rho_{1}+\rho_{2}+\ldots+\rho_{r}\right)-\left(\rho_{1}+\rho_{2}+\ldots+\rho_{p}\right) \\
& =2 m-|H| . \text { Where } m=\rho_{1}+\rho_{2}+\ldots+\rho_{r} .
\end{aligned}
$$

Therefore, $E_{H d}(G)=2 m-|H|$, and hence the proof.

\section{MINIMUM HUB DISTANCE ENERGY OF SOME STANDARD GRAPHS}

THEOREM 7. For the complete graph $K_{p}, p \geq 2$,

$$
E_{H d}\left(K_{p}\right)=2 p-2 .
$$

PROOF. Let $K_{p}$ be the complete graph with vertex set $V=$ $\left\{v_{1}, v_{2}, \cdots, v_{p}\right\}$. Then the minimum hub number is $h\left(K_{p}\right)=0$. Then

$$
A_{H d}\left(K_{p}\right)=\left(\begin{array}{ccccccccc}
0 & 1 & 1 & \cdots & 1 & 1 & \cdots & 1 & 1 \\
1 & 0 & 1 & \cdots & 1 & 1 & \cdots & 1 & 1 \\
1 & 1 & 0 & \cdots & 1 & 1 & \cdots & 1 & 1 \\
\vdots & \vdots & \vdots & \ddots & \vdots & \vdots & \ddots & \vdots & \vdots \\
1 & 1 & 1 & \cdots & 0 & 1 & \cdots & 1 & 1 \\
1 & 1 & 1 & \cdots & 1 & 0 & \cdots & 1 & 1 \\
\vdots & \vdots & \vdots & \ddots & \vdots & \vdots & \ddots & \vdots & \vdots \\
1 & 1 & 1 & \cdots & 1 & 1 & \cdots & 0 & 1 \\
1 & 1 & 1 & \cdots & 1 & 1 & \cdots & 1 & 0
\end{array}\right)_{p \times p}
$$

The respective characteristic polynomial is

$$
f_{p}\left(K_{p}, \rho\right)=\left|\begin{array}{ccccccccc}
\rho & -1 & -1 & \cdots & -1 & -1 & \cdots & -1 & -1 \\
-1 & \rho & -1 & \cdots & -1 & -1 & \cdots & -1 & -1 \\
-1 & -1 & \rho & \cdots & -1 & -1 & \cdots & -1 & -1 \\
\vdots & \vdots & \vdots & \ddots & \vdots & \vdots & \ddots & \vdots & \vdots \\
-1 & -1 & -1 & \cdots & \rho & -1 & \cdots & -1 & -1 \\
-1 & -1 & -1 & \cdots & -1 & \rho & \cdots & -1 & -1 \\
\vdots & \vdots & \vdots & \ddots & \vdots & \vdots & \ddots & \vdots & \vdots \\
-1 & -1 & -1 & \cdots & -1 & -1 & \cdots & \rho & -1 \\
-1 & -1 & -1 & \cdots & -1 & -1 & \cdots & -1 & \rho
\end{array}\right|
$$

$=(\rho-(p-1))(\rho+1)^{p-1}$.

Hence, minimum hub distance eigenvalues are $\rho=p-$ 1 [one time], $\rho=-1$ [ $p-1$ time].

Therefore, the minimum hub distance energy of a complete graph

$$
E_{H d}\left(K_{p}\right)=2 p-2 \text {. }
$$

THEOREM 8. For the complete bipartite graph $K_{n, n}, n \geq 3$, the minimum hub distance energy is $(4 n-8)+\sqrt{n^{2}-2 n+5}+$ $\sqrt{9 n^{2}-6 n+13}$.
Proof. For the complete bipartite graph $K_{n, n}, n \geq 3$ with vertex set $V=\left\{u_{1}, u_{2}, \cdots, u_{n}, v_{1}, v_{2}, \cdots, v_{n}\right\}$, consider a minimum hub set $H=\left\{u_{1}, v_{1}\right\}$. Then

$$
A_{H d}\left(K_{n, n}\right)=\left(\begin{array}{cccccccccc}
1 & 2 & 2 & \cdots & 2 & 1 & 1 & 1 & \cdots & 1 \\
2 & 0 & 2 & \cdots & 2 & 1 & 1 & 1 & \cdots & 1 \\
2 & 2 & 0 & \cdots & 2 & 1 & 1 & 1 & \cdots & 1 \\
\vdots & \vdots & \vdots & \ddots & \vdots & \vdots & \vdots & \vdots & \ddots & \vdots \\
2 & 2 & 2 & \cdots & 0 & 1 & 1 & 1 & \cdots & 1 \\
1 & 1 & 1 & \cdots & 1 & 1 & 2 & 2 & \cdots & 2 \\
1 & 1 & 1 & \cdots & 1 & 2 & 0 & 2 & \cdots & 2 \\
1 & 1 & 1 & \cdots & 1 & 2 & 2 & 0 & \cdots & 2 \\
\vdots & \vdots & \vdots & \ddots & \vdots & \vdots & \vdots & \vdots & \ddots & \vdots \\
1 & 1 & 1 & \cdots & 1 & 2 & 2 & 2 & \cdots & 0
\end{array}\right)_{(2 n) \times(2 n)}
$$

The characteristic polynomial of $A_{H d}\left(K_{n, n}\right)$,

$$
f_{2 n}\left(K_{n, n}, \rho\right)=\left|\begin{array}{cccccccccc}
\rho-1 & -2 & -2 & \cdots & -2 & -1 & -1 & -1 & \cdots & -1 \\
-2 & \rho & -2 & \cdots & -2 & -1 & -1 & -1 & \cdots & -1 \\
-2 & -2 & \rho & \cdots & -2 & -1 & -1 & -1 & \cdots & -1 \\
\vdots & \vdots & \vdots & \ddots & \vdots & \vdots & \vdots & \vdots & \ddots & \vdots \\
-2 & -2 & -2 & \cdots & \rho & -1 & -1 & -1 & \cdots & -1 \\
-1 & -1 & -1 & \cdots & -1 & \rho-1 & -2 & -2 & \cdots & -2 \\
-1 & -1 & -1 & \cdots & -1 & -2 & \rho & -2 & \cdots & -2 \\
-1 & -1 & -1 & \cdots & -1 & -2 & -2 & \rho & \cdots & -2 \\
\vdots & \vdots & \vdots & \ddots & \vdots & \vdots & \vdots & \vdots & \ddots & \vdots \\
-1 & -1 & -1 & \cdots & -1 & -2 & -2 & -2 & \cdots & \rho
\end{array}\right|
$$

$=(\rho+2)^{2 n-4}\left[\rho^{2}-(n-3) \rho-(n-1)\right]\left[\rho^{2}-(3 n-3) \rho-(3 n+1)\right]$. Hence, minimum hub distance eigenvalues are $\rho=-2[2 n-$ 4 times], $\rho=\frac{(n-3) \pm \sqrt{n^{2}-2 n+5}}{2}$ [one time each], $\rho=$ $\frac{(3 n-3) \pm \sqrt{9 n^{2}-6 n+13}}{2}$ [one time each].

Therefore, the minimum hub distance energy of a complete bipartite graph is $E_{H d}\left(K_{n, n}\right)=(4 n-8)+\sqrt{n^{2}-2 n+5}+$ $\sqrt{9 n^{2}-6 n+13}$.

THEOREM 9. For $n \geq 2$, the minimum hub distance energy of a star graph $K_{1, p-1}$ is equal to $4 p-7$.

PROOF. Let $K_{1, p-1}$ be a star graph with vertex set $V=$ $\left\{v_{0}, v_{1}, v_{2}, \cdots, v_{p-1}\right\}, v_{0}$ is the center, and the minimum hub set is $H=\left\{v_{0}\right\}$. Then

$$
A_{H d}\left(K_{1, p-1}\right)=\left(\begin{array}{ccccc}
1 & 1 & 1 & \cdots & 1 \\
1 & 0 & 2 & \cdots & 2 \\
1 & 2 & 0 & \cdots & 2 \\
\vdots & \vdots & \vdots & \ddots & \vdots \\
1 & 2 & 2 & \cdots & 0
\end{array}\right)_{p \times p}
$$

The characteristic polynomial of $A_{H d}\left(K_{1, p-1}\right)$ is

$$
\begin{aligned}
& f_{p}\left(K_{1, p-1}, \rho\right)=\left|\begin{array}{ccccc}
\rho-1 & -1 & -1 & \cdots & -1 \\
-1 & \rho & -2 & \cdots & -2 \\
-1 & -2 & \rho & \cdots & -2 \\
\vdots & \vdots & \vdots & \ddots & \vdots \\
-1 & -2 & -2 & \cdots & \rho
\end{array}\right| \\
& =(\rho+2)^{p-2}\left(\rho^{2}-(2 p-3) \rho+(p-3)\right) .
\end{aligned}
$$


Hence, minimum hub distance eigenvalues are $\rho=-2[p-$ 2 times], $\rho=\frac{(2 p-3) \pm \sqrt{4 p^{2}-16 p+21}}{2}$ [one time each]. Therefore, the minimum distance energy of a star graph is

$$
E_{H d}\left(K_{1, p-1}\right)=4 p-7 .
$$

DEFINITION 10. 87 The double star graph $S_{n, m}$ is the graph constructed from $K_{1, n-1}$ and $K_{1, m-1}$ by joining their centers $v_{0}$ and $u_{0} . V\left(S_{n, m}\right)=V\left(K_{1, n-1}\right) \cup V\left(K_{1, m-1}\right)$ and $E\left(S_{n, m}\right)=$ $\left\{v_{0} u_{0}, v_{0} v_{i}, u_{0} u_{j}: 1 \leq i \leq n-1,1 \leq j \leq m-1\right\}$. Therefore, double star graph is bipartite graph.

THEOREM 11 . For $n \geq 3$, the minimum hub distance energy of the double star graph $S_{n, n}$ is equal to $(9 n-13)+\sqrt{n^{2}+6 n-3}$.

Proof. For the double star graph $S_{n, n}$ with vertex set $V=$ $\left\{v_{0}, v_{1}, \ldots, v_{n-1}, u_{0}, u_{1}, \ldots, u_{n-1}\right\}$ the minimum hub set is $H=$ $\left\{v_{0}, u_{0}\right\}$. Then

$$
A_{H d}\left(S_{n, n}\right)=\left(\begin{array}{cccccccccc}
1 & 1 & 1 & \cdots & 1 & 1 & 2 & 2 & \cdots & 2 \\
1 & 0 & 2 & \cdots & 2 & 2 & 3 & 3 & \cdots & 3 \\
1 & 2 & 0 & \cdots & 2 & 2 & 3 & 3 & \cdots & 3 \\
\vdots & \vdots & \vdots & \ddots & \vdots & \vdots & \vdots & \vdots & \ddots & \vdots \\
1 & 2 & 2 & \cdots & 0 & 2 & 3 & 3 & \cdots & 3 \\
1 & 2 & 2 & \cdots & 2 & 1 & 1 & 1 & \cdots & 1 \\
2 & 3 & 3 & \cdots & 3 & 1 & 0 & 2 & \cdots & 2 \\
2 & 3 & 3 & \cdots & 3 & 1 & 2 & 0 & \cdots & 2 \\
\vdots & \vdots & \vdots & \ddots & \vdots & \vdots & \vdots & \vdots & \ddots & \vdots \\
2 & 3 & 3 & \cdots & 3 & 1 & 2 & 2 & \cdots & 0
\end{array}\right)_{2 n \times 2 n}
$$

The characteristic polynomial of $A_{H d}\left(S_{n, n}\right)$ is

$$
f_{2 n}\left(S_{n, n}, \rho\right)=\left|\begin{array}{cccccccccc}
\rho-1 & -1 & -1 & \cdots & -1 & -1 & -2 & -2 & \cdots & -2 \\
-1 & \rho & -2 & \cdots & -2 & -2 & -3 & -3 & \cdots & -3 \\
-1 & -2 & \rho & \cdots & -2 & -2 & -3 & -3 & \cdots & -3 \\
\vdots & \vdots & \vdots & \ddots & \vdots & \vdots & \vdots & \vdots & \ddots & \vdots \\
-1 & -2 & -2 & \cdots & \rho & -2 & -3 & -3 & \cdots & -3 \\
-1 & -2 & -2 & \cdots & -2 & \rho-1 & -1 & -1 & \cdots & -1 \\
-2 & -3 & -3 & \cdots & -3 & -1 & \rho & -2 & \cdots & -2 \\
-2 & -3 & -3 & \cdots & -3 & -1 & -2 & \rho & \cdots & -2 \\
\vdots & \vdots & \vdots & \ddots & \vdots & \vdots & \vdots & \vdots & \ddots & \vdots \\
-2 & -3 & -3 & \cdots & -3 & -1 & -2 & -2 & \cdots & \rho
\end{array}\right|
$$

$=(\rho+2)^{2 n-4}\left[\rho^{2}+(n+1) \rho-(n-1)\right]\left[\rho^{2}-(5 n-5) \rho+(n-5)\right]$. Hence, minimum hub distance eigenvalues are $\rho=-2[2 n-$ 4 times], $\rho=\frac{-n-1 \pm \sqrt{n^{2}+6 n-3}}{2}$ [one time each], $\rho=$ $\frac{(5 n-5) \pm \sqrt{25 n^{2}-54 n+24}}{2}$ [one time each].

Therefore, the minimum hub distance energy of a double star graph is

$$
E_{H d}\left(S_{n, n}\right)=(9 n-13)+\sqrt{n^{2}+6 n-3} .
$$

DEFINITION 12. [1] The cocktail party graph, denoted by $K_{2 \times p}$, is a graph having vertex set $V(G)=\bigcup_{i=1}^{p}\left\{u_{i}, v_{i}\right\}$ and edge set $E(G)=\left\{u_{i} u_{j}, v_{i} v_{j}, u_{i} v_{j}, v_{i} u_{j}: 1 \leq i<j \leq p\right\}$. i.e. $|V(G)|=2 p,|E(G)|=\frac{p^{2}-3 p}{2}$ and for every $v \in V(G), d(v)=$ $2 p-2$.
THEOREM 13. For the cocktail party graph $K_{2 \times p}$, the minimum hub distance energy is

$$
E_{H d}\left(K_{2 \times p}\right) \geq(4 p-3)+2 \sqrt{2 p+2} .
$$

PROOF. Let $K_{2 \times p}$ be the cocktail party graph, having vertex set $V\left(K_{2 \times p}\right)=\bigcup_{i=1}^{p}\left\{u_{i}, v_{i}\right\}$. Then the hub number of $K_{2 \times p}$ is $h\left(K_{2 \times p}\right)=1$. Therefore, $H=\left\{u_{1}\right\}$. Then

$$
A_{H d}\left(K_{2 \times p}\right)=\left(\begin{array}{ccccccc}
1 & 2 & 1 & 1 & \cdots & 1 & 1 \\
2 & 0 & 1 & 1 & \cdots & 1 & 1 \\
1 & 1 & 0 & 2 & \cdots & 1 & 1 \\
1 & 1 & 2 & 0 & \cdots & 1 & 1 \\
\vdots & \vdots & \vdots & \vdots & \ddots & \vdots & \vdots \\
1 & 1 & 1 & 1 & \cdots & 0 & 2 \\
1 & 1 & 1 & 1 & \cdots & 2 & 0
\end{array}\right)_{2 p \times 2 p}
$$

The characteristic polynomial of $A_{H d}\left(K_{2 \times p}\right)$ is

$$
\begin{aligned}
\quad & f_{2 p}\left(K_{2 \times p}, \rho\right)=\left|\begin{array}{ccccccc}
\rho-1 & -2 & -1 & -1 & \cdots & -1 & -1 \\
-2 & \rho & -1 & -1 & \cdots & -1 & -1 \\
-1 & -1 & \rho & -2 & \cdots & -1 & -1 \\
-1 & -1 & -2 & \rho & \cdots & -1 & -1 \\
\vdots & \vdots & \vdots & \vdots & \ddots & \vdots & \vdots \\
-1 & -1 & -1 & -1 & \cdots & \rho & -2 \\
-1 & -1 & -1 & -1 & \cdots & -2 & \rho
\end{array}\right| \\
= & \rho^{p-2}(\rho+2)^{p-1}\left(\rho^{3}-(2 p-1) \rho^{2}+(2 p+2) \rho+(2 p-2)\right) \\
= & \rho^{p-2}(\rho+2)^{p-1}\left[\left(\rho^{3}-(2 p-1) \rho^{2}+\right.\right. \\
& \left.(2 p+2) \rho+(2 p-2)-\left(4 p^{2}+2 p-2\right)\right] \\
\geq & \rho^{p-2}(\rho+2)^{p-1}\left[\rho^{2}(\rho-(2 p-1))-(2 p+2)(\rho-(2 p-1))\right] \\
= & \rho^{p-2}(\rho+2)^{p-1}\left[(\rho-(2 p-1))\left(\rho^{2}-(2 p+2)\right] .\right.
\end{aligned}
$$

Therefore, minimum hub distance eigenvalues are $\rho \approx-2[p-$ 1 times], $\rho \approx 0$ [p-2 times], $\rho \approx 2 p-1$ [one time], $\rho \approx$ $\pm \sqrt{2 p+2}$ [one time each]. Where $\approx$ represents approximately equal. Hence,

$$
E_{H d}\left(K_{2 \times p}\right) \geq(4 p-3)+2 \sqrt{2 p+2} .
$$

DEFINITION 14. A Friendship graph $F_{n}$ is a one point union of $n$ copies of cycle $C_{3} . V\left(F_{n}\right)=2 n+1$.

THEOREM 15. For the A Friendship graph $F_{n}, n \geq 2$, the minimum hub distance energy is

$$
E_{H d}\left(F_{n}\right)=8 n-5 .
$$

Proof. Let $F_{n}, n \geq 2$ be the Friendship graph, having vertex set $V\left(F_{n}\right)=\left\{v_{0}, v_{1}, \ldots \ldots ., v_{2 n}\right\}, v_{0}$ is the center, and the hub number of $F_{n}$ is $h\left(F_{n}\right)=1$. Therefore, the minimum hub set $H=$ $\left\{v_{0}\right\}$. Then

$$
A_{H d}\left(F_{n}\right)=\left(\begin{array}{cccccccc}
1 & 1 & 1 & 1 & 1 & \cdots & 1 & 1 \\
1 & 0 & 1 & 2 & 2 & \cdots & 2 & 2 \\
1 & 1 & 0 & 2 & 2 & \cdots & 2 & 2 \\
1 & 2 & 2 & 0 & 1 & \cdots & 2 & 2 \\
1 & 2 & 2 & 1 & 0 & \cdots & 2 & 2 \\
\vdots & \vdots & \vdots & \vdots & \vdots & \ddots & \vdots & \vdots \\
1 & 2 & 2 & 2 & 2 & \cdots & 0 & 1 \\
1 & 2 & 2 & 2 & 2 & \cdots & 1 & 0
\end{array}\right)_{(2 n+1) \times(2 n+1)}
$$


The characteristic polynomial of $A_{H d}\left(F_{n}\right)$ is

$$
f_{2 n+1}\left(F_{n}, \rho\right)=\left|\begin{array}{cccccccc}
\rho-1 & -1 & -1 & -1 & -1 & \cdots & -1 & -1 \\
-1 & \rho & -1 & -2 & -2 & \cdots & -2 & -2 \\
-1 & -1 & \rho & -2 & -2 & \cdots & -2 & -2 \\
-1 & -2 & -2 & \rho & -1 & \cdots & -2 & -2 \\
-1 & -2 & -2 & -1 & \rho & \cdots & -2 & -2 \\
\vdots & \vdots & \vdots & \vdots & \vdots & \ddots & \vdots & \vdots \\
-1 & -2 & -2 & -2 & -2 & \cdots & \rho & -1 \\
-1 & -2 & -2 & -2 & -2 & \cdots & -1 & \rho
\end{array}\right|
$$

$=(\rho+3)^{n-1}(\rho+1)^{n}\left[\rho^{2}-(4 n-2) \rho+(2 n-3]\right.$.

Hence, minimum hub distance eigenvalues are $\rho=$ $-3[n-1$ times $], \rho=-1 \quad[n$ times $], \rho=$ $\frac{4 n-2 \pm \sqrt{16 n^{2}-24 n+16}}{2}$ [one time each].

Therefore, the minimum hub distance energy of a friendship graph is

$$
E_{H d}\left(F_{n}\right)=(8 n-5) .
$$

\section{BOUNDS FOR MINIMUM HUB DISTANCE ENERGY OF A GRAPH}

THEOREM 16. Let $G$ be a graph of order $p$ and size $q$. Then $\sqrt{h(G)+2 q+2 \sum_{i<j, d\left(v_{i}, v_{j}\right) \neq 1} d^{2}\left(v_{i}, v_{j}\right)} \leq E_{H d}(G) \leq$ $\sqrt{p\left[h(G)+2 q+2 \sum_{i<j, d\left(v_{i}, v_{j}\right) \neq 1} d^{2}\left(v_{i}, v_{j}\right)\right]}$

PROOF. Consider the Cauchy-Schwartz inequality

$$
\left(\sum_{i=1}^{p} a_{i} b_{i}\right)^{2} \leq\left(\sum_{i=1}^{p} a_{i}^{2}\right)\left(\sum_{i=1}^{p} b_{i}^{2}\right) .
$$

By choosing $a_{i}=1$ and $b_{i}=\left|\rho_{i}\right|$, we get $\left(E_{H d}(G)\right)^{2}=\left(\sum_{i=1}^{p}\left|\rho_{i}\right|\right)^{2} \leq\left(\sum_{i=1}^{p} 1\right)\left(\sum_{i=1}^{p} \rho_{i}^{2}\right) \leq$ $p\left[h(G)+2 q+2 \sum_{i<j, d\left(v_{i}, v_{j}\right) \neq 1} d^{2}\left(v_{i}, v_{j}\right)\right]$. Therefore, the upper bound holds.

Now, since

$$
\left(\sum_{i=1}^{p}\left|\rho_{i}\right|\right)^{2} \geq \sum_{i=1}^{p} \rho_{i}^{2}
$$

we have

$$
\left(E_{H d}(G)\right)^{2} \geq \sum_{i=1}^{p} \rho_{i}^{2}=h(G)+2 q+2 \sum_{i<j, d\left(v_{i}, v_{j}\right) \neq 1} d^{2}\left(v_{i}, v_{j}\right) .
$$

Therefore.

$$
E_{H d}(G) \geq \sqrt{h(G)+2 q+2 \sum_{i<j, d\left(v_{i}, v_{j}\right) \neq 1} d^{2}\left(v_{i}, v_{j}\right)} .
$$

THEOREM 17. Let $G$ be a connected graph of order $p$ and size $q$. Then $\sqrt{2 p-q-1+2 \sum_{i<j, d\left(v_{i}, v_{j}\right) \neq 1} d^{2}\left(v_{i}, v_{j}\right)}<$ $E_{H d}(G) \leq \sqrt{p^{3}-\Delta p+2 p \sum_{i<j, d\left(v_{i}, v_{j}\right) \neq 1} d^{2}\left(v_{i}, v_{j}\right)}$.
Proof. By Lemma 2 and Theorem 3 we have

$$
\gamma(G)-1 \leq h(G) \leq p-\Delta
$$

Since for any graph, $2 q \leq p^{2}-p$, it follows by Theorem 16 that $E_{H d}(G) \leq \sqrt{p\left[h(G)+2 q+2 \sum_{i<j, d\left(v_{i}, v_{j}\right) \neq 1} d^{2}\left(v_{i}, v_{j}\right)\right]} \leq$
$\sqrt{p\left[\left(p^{2}-p\right)+p-\Delta+2 \sum_{i<j, d\left(v_{i}, v_{j}\right) \neq 1} d^{2}\left(v_{i}, v_{j}\right)\right]}=$ $\sqrt{\left[\left(p^{3}-\Delta p\right)++2 p \sum_{i<j, d\left(v_{i}, v_{j}\right) \neq 1} d^{2}\left(v_{i}, v_{j}\right)\right]}$. For the lower bound, since for any connected graph $p \leq 2 q$, by Theorem 16. Equation 1 and Theorem 11 that it follows $E_{H d}(G) \geq \sqrt{2 q+h(G)+2 \sum_{i<j, d\left(v_{i}, v_{j}\right) \neq 1} d^{2}\left(v_{i}, v_{j}\right)} \geq$ $\sqrt{p+\gamma(G)-1+2 \sum_{i<j, d\left(v_{i}, v_{j}\right) \neq 1} d^{2}\left(v_{i}, v_{j}\right)}$ $\sqrt{2 p-q-1+2 \sum_{i<j, d\left(v_{i}, v_{j}\right) \neq 1} d^{2}\left(v_{i}, v_{j}\right)}$.

THEOREM 18. Let $G$ be a graph of order and size $p$ and $q$, respectively. If $K=\operatorname{det}\left(A_{H d}(G)\right)$, then

$$
E_{H d}(G) \geq \sqrt{h(G)+2 q+2 \sum_{i<j, d\left(v_{i}, v_{j}\right) \neq 1} d^{2}\left(v_{i}, v_{j}\right)+p(p-1) K^{2 / p}} .
$$

Proof. Since $\left(E_{H d}(G)\right)^{2}=\left(\sum_{i=1}^{p}\left|\rho_{i}\right|\right)^{2}=$ $\left(\sum_{i=1}^{p}\left|\rho_{i}\right|\right)\left(\sum_{i=1}^{p}\left|\rho_{i}\right|\right)=\sum_{i=1}^{p}\left|\rho_{i}\right|^{2}+\sum_{i \neq j}\left|\rho_{i} \| \rho_{j}\right|$. using the inequality between the arithmetic and geometric means, we get

$$
\frac{1}{p(p-1)} \sum_{i \neq j}\left|\rho_{i}\right|\left|\rho_{j}\right| \geq\left(\prod_{i \neq j}\left|\rho_{i}\right|\left|\rho_{j}\right|\right)^{1 /[p(p-1)]}
$$

Thus

$$
\begin{aligned}
\left(E_{H d}(G)\right)^{2} & \geq \sum_{i=1}^{p}\left|\rho_{i}\right|^{2}+p(p-1)\left(\prod_{i \neq j}\left|\rho_{i}\right|\left|\rho_{j}\right|\right)^{1 /[p(p-1)]} \\
& \geq \sum_{i=1}^{p}\left|\rho_{i}\right|^{2}+p(p-1)\left(\prod_{i=j}\left|\rho_{i}\right|^{2(p-1)}\right)^{1 /[p(p-1)]} \\
& =\sum_{i=1}^{p}\left|\rho_{i}\right|^{2}+p(p-1)\left|\prod_{i \neq j} \rho_{i}\right|^{2 / p} \sum_{i<j, d\left(v_{i}, v_{j}\right) \neq 1} d^{2}\left(v_{i}, v_{j}\right)+p(p-1) K^{2 / p}
\end{aligned}
$$

\section{CONCLUSION}

In this paper, we obtain the bounds for minimum hub distance energy of graphs and present its exact value for complete graph, complete bipartite graph, star graph, double star graph, cocktail party graph and a friendship graph. The minimum hub distance energy of several other families of graphs is an open problem. 


\section{REFERENCES}

[1] C. Adiga, A. Bayad, I. Gutman, S. A. Srinivas, The minimum covering energy of a graph, Kragujevac Journal of Science, 34(2012), 39-56.

[2] R. B. Bapat, Graphs and Matrices, Hindustan Book Agency, 2011.

[3] R. B. Bapat, S. Pati, Energy of a graph is never an odd integer, Bulletin of Kerala Mathematics Association, 1(2011), 129-132.

[4] J. Bermond, J. Bond, D. Peleg and S. Perennes, The power of small coalitions in graphs, Discrete Applied Mathematics, 127(2003), 399 - 414.

[5] S. B. Bozkurt, A. D. Güngör and B. Zhou, Note on the distance energy of graphs, MATCH Communication in Mathematical and in Computer Chemistry, 64 (2010), 129-134.

[6] G. Caporossi, E. Chasset and B. Furtula, Some conjectures and properties on distance energy, Les Cahiers du GERAD, 64 (2009), 1-7.

[7] T. Grauman, S. Hartke, A. Jobson, B. Kinnersley, D. west, L. wiglesworth, P. Worah and $\mathrm{H}$. Wu, The hub number of a graph, Information processing letters , 108(2008), 226-228.

[8] J. W. Grossman, F. Harary, M. Klawe, Generalized ramsey theorem for graphs, X: Double stars, Discrete Mathematics, 28(1979), 247-254.

[9] I. Gutman, The energy of a graph, Ber. Math-Statist. Sekt. Forschungsz. Graz, 103(1978), 1-22.

[10] I. Gutman, X. Li, J. Zhang, Graph Energy, (Ed-s: M. Dehmer, F. Em-mert) Streib., Analysis of Complex Networks, From Biology to Linguistics, Wiley-VCH, Weinheim, (2009), 145-174.

[11] A. D. Güngör and S. B. Bozkurt, On the distance spectral radius and distance energy of graphs, Linear Multilinear Algebra, 59 (2011), 365-370.

[12] F. Harary, Graph Theory, Addison Wesley, Massachusetts, 1969.

[13] T. W. Haynes, S. T. Hedetniemi and P. J. Slater, Fundamentals of Domination in Graphs, Marcel Dckker, New York, 1998.

[14] G. Indulal, I. Gutman and A. Vijayakumar, On distance energy of graphs, MATCH Communication in Mathematical and in Computer Chemistry, 60 (2008), 461-472.

[15] V. R. Kulli, Theory of domination in graphs, Vishwa International Publications, Gulbarga, India, 2010.

[16] X. Li, Y. Shi and Gutman, Graph Energy, Springer, New York Heidelberg Dordrecht, London, 2012.

[17] H. S. Ramane, D. S. Revankar, I. Gutman, S. B. Rao, B. D. Acharya and H. B. Walikar, Bounds for the distance energy of a graph, Kragujevac Journal of Mathematics, 31 (2008), 59-68.

[18] M. Walsh, The hub number of graphs, International Journal of Mathematics and Computer Science, 1 (2006), 117-124. 